\title{
Fuzzy prime ideals redefined
}

Bingxue Yao*

\section{"Correspondence:}

yaobingxue@163.com

School of Mathematics Science, Liaocheng University, Liaocheng,

Shandong 252059, China

\begin{abstract}
In order to generalize the notions of a $(\epsilon, \in \vee q)$-fuzzy subring and various $(\in, \in \vee q)$-fuzzy ideals of a ring, a $(\lambda, \mu)$-fuzzy subring and a $(\lambda, \mu)$-fuzzy ideal of a ring are defined. The concepts of $(\lambda, \mu)$-fuzzy semiprime, prime, semiprimary and primary ideals are introduced, and the characterizations of such fuzzy ideals are obtained based on a $(\lambda, \mu)$-cut set.
\end{abstract}

MSC: $16 L 99 ; 03 E 72$

Keywords: $(\lambda, \mu)$-fuzzy subring; $(\lambda, \mu)$-fuzzy ideal; $(\lambda, \mu)$-fuzzy prime ideal; $(\lambda, \mu)$-fuzzy primary ideal

\section{Introduction}

The concept of a fuzzy set introduced by Zadeh [1] was applied to the group theory by Rosenfeld [2] and the ring theory by Liu [3]. Since then, many scholars have studied the theories of fuzzy subrings and various fuzzy prime ideals [4-6]. It is worth pointing out that Bhakat and Das introduced the concept of an $(\alpha, \beta)$-fuzzy subgroup by using the 'belongs to' relation and 'quasi-coincident with' relation between a fuzzy point and a fuzzy subset, and gave the concepts of an $(\in, \in \vee q)$-fuzzy subgroup and an $(\in, \in \vee q)$-fuzzy subring $[7,8]$. It is well known that a fuzzy subset $A$ of a group $G$ is a Rosenfeld's fuzzy subgroup if and only if $A_{t}=\{x \in G \mid A(x) \geq t\}$ is a subgroup of $G$ for all $t \in(0,1]$ (for our convenience, here $\emptyset$ is regarded as a subgroup of $G)$. Similarly, $A$ is an $(\in, \in \vee q)$-fuzzy subgroup if and only if $A_{t}$ is a subgroup of $G$ for all $t \in(0,0.5]$. A corresponding result should be considered when $A_{t}$ is a subgroup of $G$ for all $t \in(a, b]$, where $(a, b]$ is an arbitrary subinterval of [0,1]. Motivated by the above problem, Yuan et al. [9] introduced a fuzzy subgroup with the thresholds of a group. In order to generalize the concepts of an $(\in, \in \vee q)$-fuzzy subring and an $(\in, \in \vee q)$-fuzzy ideal of a ring, Yao [10] introduced the notions of a $(\lambda, \mu)$-fuzzy subring and a $(\lambda, \mu)$-fuzzy ideal and discussed their fundamental properties. In this paper, we will introduce the concepts of $(\lambda, \mu)$-fuzzy prime, fuzzy semiprime, fuzzy primary and fuzzy semiprimary ideals of a ring.

\section{$2(\lambda, \mu)$-fuzzy ideal}

Let $X$ be a nonempty set. By a fuzzy subset $A$ of $X$, we mean a map from $X$ to the interval $[0,1], A: X \rightarrow[0,1]$. If $A$ is a fuzzy subset of $X$ and $t \in[0,1]$, then the cut set $A_{t}$ and the open cut set $A_{\langle t\rangle}$ of $A$ are defined as follows:

$$
A_{t}=\{x \in X \mid A(x) \geq t\}, \quad A_{\langle t\rangle}=\{x \in X \mid A(x)>t\} .
$$

First, we recall some definitions and results for the sake of completeness.

2012 Yao; licensee Springer. This is an Open Access article distributed under the terms of the Creative Commons Attribution License (http://creativecommons.org/licenses/by/2.0), which permits unrestricted use, distribution, and reproduction in any medium, provided the original work is properly cited. 
Definition 1 Let $x \in X, t \in(0,1]$. A fuzzy subset $A$ of $X$ of the form

$$
A(y)= \begin{cases}t, & \text { if } y=x, \\ 0, & \text { if } y \neq x\end{cases}
$$

is said to be a fuzzy point with support $x$ and value $t$ and is denoted by $x_{t}$.

Definition 2 [11] A fuzzy point $x_{t}$ is said to belong to (resp. be quasi-coincident with) a fuzzy subset $A$, written as $x_{t} \in A$ (resp. $\left.x_{t} q A\right)$, if

$$
A(x) \geq t \quad(\text { resp. } A(x)+t>1)
$$

$x_{t} \in A$ or $x_{t} q A$ will be denoted by $x_{t} \in \vee q A$.

In the following discussions, $R$ always stands for an associate ring, $\lambda$ and $\mu$ are constant numbers such that $0 \leq \lambda<\mu \leq 1$, and $N$ denotes the set of all positive integers.

Definition 3 [8] A fuzzy subset $A$ of $R$ is said to be an $(\in, \in \vee q)$-fuzzy subring of $R$ if for all $x, y \in R$ and $t, r \in(0,1]$,

(1) $x_{t}, y_{r} \in A \Longrightarrow(x+y)_{t \wedge r} \in \vee q A$,

(2) $x_{t} \in A \Longrightarrow(-x)_{t} \in \vee q A$,

(3) $x_{t}, y_{r} \in A \Longrightarrow(x y)_{t \wedge r} \in \vee q A$.

Definition 4 [8] A fuzzy subset $A$ of $R$ is said to be an $(\in, \in \vee q)$-fuzzy ideal of $R$ if

(1) $A$ is an $(\in, \in \vee q)$-fuzzy subring of $R$,

(2) $x_{t} \in A, y \in R \Longrightarrow(x y)_{t},(y x)_{t} \in \vee q A, \forall t \in(0,1]$.

According to Definition 3 and Definition 4, we have that a fuzzy subset $A$ of $R$ is a $(\lambda, \mu)$ fuzzy subring (ideal) of $R$ if and only if for all $x, y \in R$,

(1) $A(x-y) \geq A(x) \wedge A(y) \wedge 0.5$,

(2) $A(x y) \geq A(x) \wedge A(y) \wedge 0.5\left((2)^{\prime} A(x y) \geq(A(x) \vee A(y)) \wedge 0.5\right)$.

In order to give more general concepts of a fuzzy subring and a fuzzy ideal of $R$, we introduce the following definitions.

Definition 5 A fuzzy subset $A$ of $R$ is said to be a $(\lambda, \mu)$-fuzzy addition subgroup of $R$ if for all $x, y \in R$,

$$
A(x+y) \vee \lambda \geq A(x) \wedge A(y) \wedge \mu, \quad A(-x) \vee \lambda \geq A(x) \wedge \mu .
$$

Clearly, a fuzzy subset $A$ of $R$ is a $(\lambda, \mu)$-fuzzy addition subgroup of $R$ if and only if for all $x, y \in R, A(x-y) \vee \lambda \geq A(x) \wedge A(y) \wedge \mu$.

Definition 6 [10] A fuzzy subset $A$ of $R$ is said to be a $(\lambda, \mu)$-fuzzy subring of $R$ if for all $x, y \in R$,

(1) $A(x-y) \vee \lambda \geq A(x) \wedge A(y) \wedge \mu$,

(2) $A(x y) \vee \lambda \geq A(x) \wedge A(y) \wedge \mu$. 
Definition 7 [10] A fuzzy subset $A$ of $R$ is said to be a $(\lambda, \mu)$-fuzzy left ideal (resp. fuzzy right ideal) of $R$ if for all $x, y \in R$,

(1) $A(x-y) \vee \lambda \geq A(x) \wedge A(y) \wedge \mu$,

(2) $A(x y) \vee \lambda \geq A(y) \wedge \mu($ resp. $A(x y) \vee \lambda \geq A(x) \wedge \mu)$.

$A$ is said to be a $(\lambda, \mu)$-fuzzy ideal of $R$ if it is both a $(\lambda, \mu)$-fuzzy left ideal and a $(\lambda, \mu)$-fuzzy right ideal of $R$.

According to the above definitions, a $(\lambda, \mu)$-fuzzy left ideal or a $(\lambda, \mu)$-fuzzy right ideal of $R$ must be a $(\lambda, \mu)$-fuzzy subring. A fuzzy subset $A$ of $R$ is a $(\lambda, \mu)$-fuzzy ideal of $R$ if and only if for all $x, y \in R$,

(1) $A(x-y) \vee \lambda \geq A(x) \wedge A(y) \wedge \mu$,

(2) $A(x y) \vee \lambda \geq(A(x) \vee A(y)) \wedge \mu$.

Obviously, an $(\in, \in \vee q)$-fuzzy subring (fuzzy ideal) of $R$ is a $(\lambda, \mu)$-fuzzy subring (fuzzy ideal) of $R$ with $\lambda=0$ and $\mu=0.5$.

The following theorem is obvious.

Theorem 1 Let $A, B$ be ( $\lambda, \mu)$-fuzzy left ideals (fuzzy right ideals, fuzzy ideals, fuzzy subrings) of $R$. Then $A \cap B$ is also a fuzzy left ideal (fuzzy right ideal, fuzzy ideal, fuzzy subring) of $R$.

Theorem 2 Let $A, B$ be $(\lambda, \mu)$-fuzzy left ideals (fuzzy right ideals, fuzzy ideals) of $R$. Then $A+B$ is also a $(\lambda, \mu)$-fuzzy left ideal (fuzzy right ideal, fuzzy ideal) of $R$, where

$$
(A+B)(x)=\sup \left\{A\left(x_{1}\right) \wedge B\left(x_{2}\right) \mid x=x_{1}+x_{2}\right\}, \quad \forall x \in R .
$$

Proof We only prove the case of a $(\lambda, \mu)$-fuzzy left ideal.

For all $x, y \in R$, we have

$$
\begin{aligned}
(A & +B)(x-y) \vee \lambda \\
& \geq \sup \left\{A\left(x_{1}-x_{2}\right) \wedge B\left(y_{1}-y_{2}\right) \mid x=x_{1}+y_{1}, y=x_{2}+y_{2}\right\} \vee \lambda \\
& \geq \sup \left\{A\left(x_{1}\right) \wedge A\left(x_{2}\right) \wedge B\left(y_{1}\right) \wedge B\left(y_{2}\right) \wedge \mu \mid x=x_{1}+y_{1}, y=x_{2}+y_{2}\right\} \\
& =\sup \left\{A\left(x_{1}\right) \wedge B\left(y_{1}\right) \mid x=x_{1}+y_{1}\right\} \wedge \sup \left\{A\left(x_{2}\right) \wedge B\left(y_{2}\right) \mid y=x_{2}+y_{2}\right\} \wedge \mu \\
& =(A+B)(x) \wedge(A+B)(y) \wedge \mu, \\
(A & +B)(x y) \vee \lambda \\
& \geq \sup \left\{A\left(x y_{1}\right) \wedge B\left(x y_{2}\right) \mid y=y_{1}+y_{2}\right\} \vee \lambda \\
& =\sup \left\{\left(A\left(x y_{1}\right) \vee \lambda\right) \wedge\left(B\left(x y_{2}\right) \vee \lambda\right) \mid y=y_{1}+y_{2}\right\} \\
& \geq \sup \left\{\left(A\left(y_{1}\right) \wedge \mu\right) \wedge\left(B\left(y_{2}\right) \wedge \mu\right) \mid y=y_{1}+y_{2}\right\} \\
& =\sup \left\{A\left(y_{1}\right) \wedge B\left(y_{2}\right) \mid y=y_{1}+y_{2}\right\} \wedge \mu \\
& =(A+B)(y) \wedge \mu .
\end{aligned}
$$

So, $A+B$ is a $(\lambda, \mu)$-fuzzy left ideal of $R$. 
Let $A, B$ be fuzzy subsets of $R$. Then the fuzzy subset $A \odot B$ is defined as follows: $\forall x \in R$,

$$
(A \odot B)(x)=\left\{\begin{aligned}
\sup \left\{\inf _{1 \leq i \leq n}\left(A\left(x_{i}\right) \wedge B\left(y_{i}\right)\right) \mid x=\sum_{i=1}^{n} x_{i} y_{i}, x_{i}, y_{i} \in R, n \in N\right\} & \\
& \text { if } x \text { can be expressed as } x=\sum x_{i} y_{i}, x_{i}, y_{i} \in R, \\
0, & \text { otherwise. }
\end{aligned}\right.
$$

Theorem 3 Let $A$ be a $(\lambda, \mu)$-fuzzy left ideal, and let $B$ be a fuzzy subset of $R$. Then $A \odot B$ is a $(\lambda, \mu)$-fuzzy left ideal of $R$.

Proof For all $z_{1}, z_{2} \in R$, we have

$$
\begin{aligned}
(A \odot B)\left(z_{1}-z_{2}\right) \vee \lambda & \sup \left\{\inf _{1 \leq i \leq n, 1 \leq j \leq m} A\left(x_{i}\right) \wedge B\left(y_{i}\right) \wedge A\left(-x_{j}^{\prime}\right) \wedge B\left(y_{j}^{\prime}\right) \mid,\right. \\
& \left.z_{1}=\sum_{i=1}^{n} x_{i} y_{i}, z_{2}=\sum_{j=1}^{m} x_{j}^{\prime} y_{j}^{\prime}, m, n \in N\right\} \vee \lambda \\
\geq & \sup \left\{\inf _{1 \leq i \leq n} A\left(x_{i}\right) \wedge B\left(y_{i}\right) \mid z_{1}=\sum_{i=1}^{n} x_{i} y_{i}, n \in N\right\} \\
& \wedge \sup \left\{\inf _{1 \leq j \leq m} A\left(x_{j}^{\prime}\right) \wedge B\left(y_{j}^{\prime}\right) \mid z_{2}=\sum_{j=1}^{m} x_{j}^{\prime} y_{j}^{\prime}, m \in N\right\} \wedge \mu \\
= & (A \odot B)\left(z_{1}\right) \wedge(A \odot B)\left(z_{2}\right) \wedge \mu, \\
(A \odot & B)\left(z_{1} z_{2}\right) \vee \lambda \geq \sup \left\{\inf _{1 \leq i \leq n} A\left(z_{1} x_{i}\right) \wedge B\left(y_{i}\right) \mid z_{2}=\sum_{i=1}^{n} x_{i} y_{i}, n \in N\right\} \vee \lambda \\
\geq & \sup \left\{\inf _{1 \leq i \leq n} A\left(x_{i}\right) \wedge B\left(y_{i}\right) \mid z_{2}=\sum_{i=1}^{n} x_{i} y_{i}, n \in N\right\} \wedge \mu \\
= & (A \odot B)\left(z_{2}\right) \wedge \mu .
\end{aligned}
$$

So, $A \odot B$ is a $(\in, \in \vee q)$-fuzzy left ideal of $R$.

Similarly, we have the following theorem.

Theorem 4 Let $A$ be a fuzzy subset, and let $B$ be a $(\lambda, \mu)$-fuzzy right ideal of $R$. Then $A \odot B$ is a $(\lambda, \mu)$-fuzzy right ideal of $R$.

The following theorem is an immediate consequence of Theorem 3 and Theorem 4.

Theorem 5 Let $A$ be a $(\lambda, \mu)$-fuzzy left ideal, and let $B$ be a $(\lambda, \mu)$-fuzzy right ideal of $R$. Then $A \odot B$ is a $(\lambda, \mu)$-fuzzy ideal of $R$.

One of the most common methods of studying a fuzzy subring and a fuzzy ideal is by using their cut sets. Now we give the relation between a $(\lambda, \mu)$-fuzzy subring (fuzzy ideal) with its cut set or open cut set. 
Theorem 6 [10] A fuzzy subset $A$ of $R$ is a $(\lambda, \mu)$-fuzzy subring (fuzzy ideal) of $R$ if and only iffor all $t \in(\lambda, \mu], A_{t}$ is a subring (ideal) of $R$ or $A_{t}=\emptyset$.

Theorem 7 A fuzzy subset $A$ of $R$ is a $(\lambda, \mu)$-fuzzy subring (fuzzy ideal) of $R$ if and only if for all $t \in[\lambda, \mu), A_{\langle t\rangle}$ is a subring (ideal) of $R$ or $A_{\langle t\rangle}=\emptyset$.

Proof We only prove the case of a $(\lambda, \mu)$-fuzzy subring.

Let $A$ be a $(\lambda, \mu)$-fuzzy subring of $R$, and let $t \in[\lambda, \mu)$. If $x, y \in A_{\langle t\rangle}$, then $A(x) \wedge A(y)>t$, $A(x-y) \vee \lambda \geq A(x) \wedge A(y) \wedge \mu>t$. Considering $\lambda \leq t$, we have $A(x-y)>t$ and $x-y \in A_{\langle t\rangle}$. Similarly, $x y \in A_{\langle t\rangle}$. It follows that $A_{\langle t\rangle}$ is a subring of $R$.

Conversely, assume that $A_{\langle t\rangle}$ is a subring of $R$ for all $t \in[\lambda, \mu)$. If possible, let $A\left(x_{0}-\right.$ $\left.y_{0}\right) \vee \lambda<A\left(x_{0}\right) \wedge A\left(y_{0}\right) \wedge \mu$ for some $x_{0}, y_{0} \in R$. Put $t=A\left(x_{0}-y_{0}\right) \vee \lambda$, then $t \in[\lambda, \mu)$, and $A\left(x_{0}-y_{0}\right) \leq t, A\left(x_{0}\right) \wedge A\left(y_{0}\right)>t$. So, $x_{0}, y_{0} \in A_{\langle t\rangle}$, and $x_{0}-y_{0} \notin A_{\langle t\rangle}$. This is a contradiction to the fact that $A_{\langle t\rangle}$ is a subring of $R$. This shows that $A(x-y) \vee \lambda \geq A(x) \wedge A(y) \wedge \mu$ holds for all $x, y \in R$.

Similarly, $A(x y) \vee \lambda \geq A(x) \wedge A(y) \wedge \mu, \forall x, y \in R$. That is, $A_{\langle t\rangle}$ is a subring of $R$.

In the following theorems, it is shown that the homomorphism image (preimage) of a $(\lambda, \mu)$-fuzzy subring is also a $(\lambda, \mu)$-fuzzy subring. Similar result can be obtained for a $(\lambda, \mu)$-fuzzy ideal under some conditions.

Theorem 8 [10] Let $f: R \rightarrow R^{\prime}$ be a homomorphism of rings. If $A$ is a $(\lambda, \mu)$-fuzzy subring of $R$, then $f(A)$ is a $(\lambda, \mu)$-fuzzy subring of $R^{\prime}$. Particularly, if $A$ is a $(\lambda, \mu)$-fuzzy ideal of $R$ and $f$ is onto, then $f(A)$ is a $(\lambda, \mu)$-fuzzy ideal of $R^{\prime}$, where

$$
f(A)(y)=\left\{\begin{array}{ll}
\sup \{A(x) \mid f(x)=y\}, & \text { iff } f^{-1}(y) \neq \emptyset, \\
0, & \text { otherwise }
\end{array} \quad \forall y \in R^{\prime} .\right.
$$

Theorem 9 [10] Let $f: R \rightarrow R^{\prime}$ be a homomorphism of rings. If $B$ is a $(\lambda, \mu)$-fuzzy subring (fuzzy ideal) of $R^{\prime}$, then $f^{-1}(B)$ is a $(\lambda, \mu)$-fuzzy subring (fuzzy ideal) of $R$, where

$$
f^{-1}(B)(x)=B(f(x)), \quad \forall x \in R .
$$

\section{$3(\lambda, \mu)$-cut set}

Based on the notion of an $(\in, \in \vee q)$-level subset defined in [12], we introduce the concept of a $(\lambda, \mu)$-cut set of a fuzzy subset. Let $A$ be a fuzzy subset of a set $X$ and $t \in[0,1]$. Then the subset $A_{t}^{(\lambda, \mu)}$ of $X$ defined by

$$
A_{t}^{(\lambda, \mu)}=\{x \in X \mid A(x) \vee \lambda \geq t \wedge \mu \text { or } A(x)>(2 \mu-t) \vee \lambda\}
$$

is said to be a $(\lambda, \mu)$-cut set of $A$. We denote $x_{t} \in_{(\lambda, \mu)} A$ if $x \in A_{t}^{(\lambda, \mu)}$.

Obviously, if $A$ is a fuzzy subset of $X$ and $t \in[0,1]$, then

$$
A_{t}^{(\lambda, \mu)}= \begin{cases}X, & t \leq \lambda, \\ A_{t}, & \lambda<t \leq \mu, \\ A_{\langle(2 \mu-t) \vee \lambda\rangle}, & t>\mu .\end{cases}
$$


Moreover, $x_{t} \in \vee q A$ coincides with $x_{t} \in_{(0,0.5)} A$, and $x_{t} \in \vee q_{k} A$ [12] coincides with $x_{t} \in_{\left(0, \frac{k}{2}\right)} A$.

Lemma 1 Let $A, B$ be fuzzy subsets of $X$. Then for all $t \in[0,1]$,

$$
A \subseteq B \quad \Longrightarrow \quad A_{t}^{(\lambda, \mu)} \subseteq B_{t}^{(\lambda, \mu)}
$$

Proof The proof is straightforward.

Theorem 10 Let $A, B$ be fuzzy subsets of $X$ and $t \in[0,1]$. Then

(1) $(A \cap B)_{t}^{(\lambda, \mu)}=A_{t}^{(\lambda, \mu)} \cap B_{t}^{(\lambda, \mu)}$

(2) $(A \cup B)_{t}^{(\lambda, \mu)}=A_{t}^{(\lambda, \mu)} \cup B_{t}^{(\lambda, \mu)}$.

\section{Proof}

(1) Obviously, we have $(A \cap B)_{t}^{(\lambda, \mu)} \subseteq A_{t}^{(\lambda, \mu)} \cap B_{t}^{(\lambda, \mu)}$ from Lemma 1. If $x \in A_{t}^{(\lambda, \mu)} \cap B_{t}^{(\lambda, \mu)}$, then $x \in A_{t}^{(\lambda, \mu)}$ and $x \in B_{t}^{(\lambda, \mu)}$. So, we have the following four cases.

Case 1, if $A(x) \vee \lambda \geq t \wedge \mu$ and $B(x) \vee \lambda \geq t \wedge \mu$, then $(A \cap B)(x) \vee \lambda \geq t \wedge \mu$. So, $x \in$ $(A \cap B)_{t}^{(\lambda, \mu)}$.

Case 2, if $A(x)>(2 \mu-t) \vee \lambda$ and $B(x)>(2 \mu-t) \vee \lambda$, then $(A \cap B)(x)>(2 \mu-t) \vee \lambda$. So, $x \in(A \cap B)_{t}^{(\lambda, \mu)}$.

Case 3, if $A(x) \vee \lambda \geq t \wedge \mu$ and $B(x)>(2 \mu-t) \vee \lambda$, then when $t \leq \mu$, we have $B(x) \vee$ $\lambda>(2 \mu-t) \vee \lambda \geq \mu \geq t \wedge \mu$ and hence $(A \cap B)(x) \vee \lambda \geq t \wedge \mu$. When $t>\mu$, we have $A(x) \geq \mu>(2 \mu-t) \vee \lambda$ and hence $(A \cap B)(x)>(2 \mu-t) \vee \lambda$. It means that $x \in(A \cap B)_{t}^{(\lambda, \mu)}$.

Case 4 , if $A(x)>(2 \mu-t) \vee \lambda$ and $B(x) \vee \lambda \geq t \wedge \mu$, then we can also obtain that $x \in$ $(A \cap B)_{t}^{(\lambda, \mu)}$ just as in Case 3 .

Therefore, $(A \cap B)_{t}^{(\lambda, \mu)}=A_{t}^{(\lambda, \mu)} \cap B_{t}^{(\lambda, \mu)}$.

(2) Obviously, we have $(A \cup B)_{t}^{(\lambda, \mu)} \supseteq A_{t}^{(\lambda, \mu)} \cup B_{t}^{(\lambda, \mu)}$ from Lemma 1 .

Let $x \in(A \cup B)_{t}^{(\lambda, \mu)}$, then either $A(x) \vee B(x) \vee \lambda \geq t \wedge \mu$ or $A(x) \vee B(x)>(2 \mu-t) \vee \lambda$. It means that $A(x) \vee \lambda \geq t \wedge \mu$, or $B(x) \vee \lambda \geq t \wedge \mu$, or $A(x)>(2 \mu-t) \vee \lambda$, or $B(x)>(2 \mu-t) \vee \lambda$. So $x \in A_{t}^{(\lambda, \mu)}$ or $x \in B_{t}^{(\lambda, \mu)}$. That is, $x \in A_{t}^{(\lambda, \mu)} \cup B_{t}^{(\lambda, \mu)}$. Hence, $(A \cup B)_{t}^{(\lambda, \mu)}=A_{t}^{(\lambda, \mu)} \cup B_{t}^{(\lambda, \mu)}$.

Theorem 11 Let $A, B, C$ be fuzzy subsets of $X$ and $t \in[0,1]$. Then

(1) $[A \cap(B \cup C)]_{t}^{(\lambda, \mu)}=(A \cap B)_{t}^{(\lambda, \mu)} \cup(A \cap C)_{t}^{(\lambda, \mu)}$,

(2) $[A \cup(B \cap C)]_{t}^{(\lambda, \mu)}=(A \cup B)_{t}^{(\lambda, \mu)} \cap(A \cup C)_{t}^{(\lambda, \mu)}$.

Proof The proof can be obtained immediately from Theorem 10.

Theorem 12 Let $A$ be a $(\lambda, \mu)$-fuzzy subring (fuzzy ideal) of $R$. Then for all $t \in[0,1], A_{t}^{(\lambda, \mu)}$ is a subring (ideal) of $R$ or $A_{t}^{(\lambda, \mu)}=\emptyset$.

Proof We only prove the case of a $(\lambda, \mu)$-fuzzy subring.

If $t \leq \lambda$, then $A_{t}^{(\lambda, \mu)}=R$. If $\lambda<t \leq \mu$, then $A_{t}^{(\lambda, \mu)}=A_{t}$ and $A_{t}$ is a subring of $R$ from Theorem 6. If $t>\mu$, then $A_{t}^{(\lambda, \mu)}=A_{\langle(2 \mu-t) \vee \lambda\rangle}$ and $(2 \mu-t) \vee \lambda \in[\lambda, \mu)$. So, $A_{t}^{(\lambda, \mu)}$ is a subring of $R$ from Theorem 7 .

Theorem 13 Let $A$ be a fuzzy subset of $R$. If for all $t \in(\lambda, \mu], A_{t}^{(\lambda, \mu)}$ is a subring (ideal) of $R$ or $A_{t}^{(\lambda, \mu)}=\emptyset$, then $A$ is a $(\lambda, \mu)$-fuzzy subring (fuzzy ideal) of $R$. 
Proof The proof can be obtained from Theorem 6 .

\section{$4(\lambda, \mu)$-fuzzy prime and fuzzy primary ideal}

There are several deferent definitions of a fuzzy prime ideal and a fuzzy primary ideal of $R$. In this section, by a prime ideal $S$ of $R$, we mean an ideal of $R$ such that $a b \in S \Longrightarrow a \in S$ or $b \in S$.

Bhakat and Das [8] defined fuzzy prime, fuzzy semiprime, fuzzy primary and fuzzy semiprimary ideals in a ring which must be $(\in, \in \vee q)$-fuzzy ideals first.

Definition 8 [8] An $(\in, \in \vee q)$-fuzzy ideal $A$ of $R$ is said to be

(1) fuzzy semiprime if for all $x \in R$ and $t \in(0,1],\left(x^{2}\right)_{t} \in A \Longrightarrow x_{t} \in \vee q A$,

(2) fuzzy prime if for all $x, y \in R$ and $t \in(0,1],(x y)_{t} \in A \Longrightarrow x_{t} \in \vee q A$ or $y_{t} \in \vee q A$,

(3) fuzzy semiprimary if for all $x, y \in R$ and $t \in(0,1],(x y)_{t} \in A \Longrightarrow x_{t}^{m} \in \vee q A$ or $y_{t}^{n} \in \vee q A$ for some $m, n \in N$,

(4) fuzzy primary if for all $x, y \in R$ and $t \in(0,1],(x y)_{t} \in A \Longrightarrow x_{t} \in \vee q A$ or $y_{t}^{m} \in \vee q A$ for some $m \in N$.

In order to generalize these notions, we introduce $(\lambda, \mu)$-fuzzy prime, $(\lambda, \mu)$-fuzzy semiprime, $(\lambda, \mu)$-fuzzy primary and $(\lambda, \mu)$-fuzzy semiprimary ideals.

Definition 9 A $(\lambda, \mu)$-fuzzy ideal $A$ of $R$ is said to be

(1) $(\lambda, \mu)$-fuzzy semiprime if for all $x \in R$ and $t \in(0,1],\left(x^{2}\right)_{t} \in A \Longrightarrow x_{t} \in(\lambda, \mu) A$,

(2) $(\lambda, \mu)$-fuzzy prime if for all $x, y \in R$ and $t \in(0,1],(x y)_{t} \in A \Longrightarrow x_{t} \in(\lambda, \mu) A$ or $y_{t} \in(\lambda, \mu) A$,

(3) ( $\lambda, \mu$ )-fuzzy semiprimary if for all $x, y \in R$ and $t \in(0,1],(x y)_{t} \in A \Longrightarrow x_{t}^{m} \in(\lambda, \mu) A$ or $y_{t}^{n} \in(\lambda, \mu) A$ for some $m, n \in N$,

(4) $(\lambda, \mu)$-fuzzy primary if for all $x, y \in R$ and $t \in(0,1],(x y)_{t} \in A \Longrightarrow x_{t} \in(\lambda, \mu) \cdot A$ or $y_{t}^{m} \in_{(\lambda, \mu)} A$ for some $m \in N$.

In the following four theorems, we give the equivalence condition of a $(\lambda, \mu)$-fuzzy prime (semiprime, primary, semiprimary) ideal.

Theorem $14 A(\lambda, \mu)$-fuzzy ideal $A$ of $R$ is $(\lambda, \mu)$-fuzzy prime if and only iffor all $x, y \in R$,

$$
\lambda \vee A(x) \vee A(y) \geq A(x y) \wedge \mu .
$$

Proof Let $A$ be $(\lambda, \mu)$-fuzzy prime. If possible, let $\lambda \vee A\left(x_{0}\right) \vee A\left(y_{0}\right)<A\left(x_{0} y_{0}\right) \wedge \mu$ for some $x_{0}, y_{0} \in R$. Put $t=A\left(x_{0} y_{0}\right) \wedge \mu$, then $A\left(x_{0}\right) \vee A\left(y_{0}\right)<t$ and $\left(x_{0} y_{0}\right)_{t} \in A$. So, $\lambda \vee A\left(x_{0}\right)<t=$ $t \wedge \mu$ and $\lambda \vee A\left(y_{0}\right)<t=t \wedge \mu$. From $\lambda \vee A\left(x_{0}\right)<t \wedge \mu$, we have $A\left(x_{0}\right) \leq \lambda \vee A\left(x_{0}\right)<t \leq$ $\mu \leq 2 \mu-t$ and hence $x_{0} \notin A_{t}^{(\lambda, \mu)}$. Similarly, $y_{0} \notin A_{t}^{(\lambda, \mu)}$. This is a contradiction. Therefore, for all $x, y \in R$, we have $\lambda \vee A(x) \vee A(y) \geq A(x y) \wedge \mu$.

Conversely, if for all $x, y \in R, \lambda \vee A(x) \vee A(y) \geq A(x y) \wedge \mu$ and $(x y)_{t} \in A$, then $\lambda \vee A(x) \vee$ $A(y) \geq t \wedge \mu$. So, $\lambda \vee A(x) \geq t \wedge \mu$ or $\lambda \vee A(y) \geq t \wedge \mu$. That is, $x \in_{(\lambda, \mu)} A$ or $y \in_{(\lambda, \mu)} A$. Hence, $\mathrm{A}$ is $(\lambda, \mu)$-fuzzy prime.

Theorem $15 A(\lambda, \mu)$-fuzzy ideal $A$ of $R$ is $(\lambda, \mu)$-fuzzy semiprime if and only iffor all $x \in R$,

$$
\lambda \vee A(x) \geq A\left(x^{2}\right) \wedge \mu \text {. }
$$


Proof The proof is similar to that of Theorem 14.

Theorem $16 A(\lambda, \mu)$-fuzzy ideal $A$ of $R$ is $(\lambda, \mu)$-fuzzy primary if and only iffor all $x, y \in R$, $\exists m_{0} \in N$ such that

$$
\lambda \vee A(x) \vee A\left(y^{m_{0}}\right) \geq A(x y) \wedge \mu .
$$

Proof Let $A$ be $(\lambda, \mu)$-fuzzy primary. If possible, there exist $x_{0}, y_{0} \in R$ such that $\lambda \vee A\left(x_{0}\right) \vee$ $A\left(y_{0}^{m}\right)<A\left(x_{0} y_{0}\right) \wedge \mu$ for all $m \in N$, then $\lambda \vee A\left(x_{0}\right) \vee A\left(y_{0}^{m}\right)<t \leq \mu$ and $\left(x_{0} y_{0}\right)_{t} \in A$, where $t=A\left(x_{0} y_{0}\right) \wedge \mu$. So, $\lambda \vee A\left(x_{0}\right)<t=t \wedge \mu$ and $\lambda \vee A\left(y_{0}^{m}\right)<t=t \wedge \mu$. From $\lambda \vee A\left(x_{0}\right)<t$, we have $A\left(x_{0}\right) \leq \lambda \vee A\left(x_{0}\right)<t \leq \mu \leq 2 \mu-t$ and hence $x_{0} \notin A_{t}^{(\lambda, \mu)}$. Similarly, $y_{0}^{m} \notin A_{t}^{(\lambda, \mu)}$. This is a contradiction. Therefore, for all $x, y \in R, \exists m_{0} \in N$ such that $\lambda \vee A(x) \vee A\left(y^{m_{0}}\right) \geq A(x y) \wedge \mu$.

Conversely, if for all $x, y \in R, \exists m_{0} \in N$ such that $\lambda \vee A(x) \vee A\left(y^{m_{0}}\right) \geq A(x y) \wedge \mu$, then from $(x y)_{t} \in A$, we have $\lambda \vee A(x) \vee A\left(y^{m_{0}}\right) \geq t \wedge \mu$. So, $\lambda \vee A(x) \geq t \wedge \mu$ or $\lambda \vee A\left(y^{m_{0}}\right) \geq t \wedge \mu$. That is, $x_{t} \in_{(\lambda, \mu)} A$ or $y_{t}^{m_{0}} \in \in_{(\lambda, \mu)} A$. Hence, $\mathrm{A}$ is $(\lambda, \mu)$-fuzzy primary.

Theorem $17 A(\lambda, \mu)$-fuzzy ideal $A$ of $R$ is $(\lambda, \mu)$-fuzzy semiprimary if and only if for all $x, y \in R, \exists m_{0}, n_{0} \in N$ such that

$$
\lambda \vee A\left(x^{m_{0}}\right) \vee A\left(y^{n_{0}}\right) \geq A(x y) \wedge \mu .
$$

Proof The proof is similar to that of Theorem 16.

Now, we characterize the $(\lambda, \mu)$-fuzzy prime (semiprimary) ideal by using its cut set.

Theorem 18 A fuzzy subset $A$ of $R$ is a $(\lambda, \mu)$-fuzzy prime (fuzzy semiprime) ideal if and only if for all $t \in(\lambda, \mu], A_{t}$ is a prime (semiprime) ideal of $R$ or $A_{t}=\emptyset$.

Proof We only prove the case of a $(\lambda, \mu)$-fuzzy prime ideal.

Let $A$ be a $(\lambda, \mu)$-fuzzy prime ideal of $R$. Then $A$ is a $(\lambda, \mu)$-fuzzy ideal of $R$. So, $A_{t}$ is an ideal of $R$ or $A_{t}=\emptyset$ from Theorem 6. For all $t \in(\lambda, \mu]$, if $x y \in A_{t}$, then $\lambda \vee A(x) \vee A(y) \geq$ $A(x y) \wedge \mu \geq t \wedge \mu=t$. Considering $\lambda<t$, we have $A(x) \vee A(y) \geq t$. It follows that $x \in A_{t}$ or $y \in A_{t}$. Hence, $A_{t}$ is a prime ideal of $R$.

Conversely, assume $A_{t}$ is a prime ideal of $R$ for all $t \in(\lambda, \mu]$ whenever $A_{t} \neq \emptyset$, then $A_{t}$ is an ideal of $R$, and hence $A$ is a $(\lambda, \mu)$-fuzzy ideal from Theorem 6. Let $t \in(0,1]$ and $(x y)_{t} \in A$. If $t \leq \lambda$, then it is clear that $x_{t} \in_{(\lambda, \mu)} A$. If $t \in(\lambda, \mu]$, then $x \in A_{t}$ or $y \in A_{t}$ since $A_{t}$ is a prime ideal of $R$. So, $x_{t} \in_{(\lambda, \mu)} A$ or $y_{t} \in_{(\lambda, \mu)} A$. If $t>\mu$, then $x y \in A_{\mu}$. It implies that $x \in A_{\mu}$ or $y \in A_{\mu}$, since $A_{\mu}$ is a prime ideal of $R$. Furthermore, we have

$$
x \in A_{\mu} \Longrightarrow A(x) \geq \mu=t \wedge \mu \Longrightarrow x \in A_{t}^{(\lambda, \mu)} \Longrightarrow x_{t} \in_{(\lambda, \mu)} A .
$$

Similarly,

$$
y \in A_{\mu} \Longrightarrow y_{t} \in_{(\lambda, \mu)} A \text {. }
$$

It follows that $A$ is a $(\lambda, \mu)$-fuzzy prime ideal of $R$. 
Theorem 19 Let $A$ be $a(\lambda, \mu)$-fuzzy ideal of $R$ such that $A \mu \neq \emptyset$, and let $B$ be $a(\lambda, \mu)$-fuzzy prime ideal of $A_{\mu}$. Then $A \cap B$ is a $(\lambda, \mu)$-fuzzy prime ideal of $A_{\mu}$.

Proof From Theorem 1 and Theorem $6, A_{\mu}$ is a subring of $R$ and $A \cap B$ is a $(\lambda, \mu)$-fuzzy ideal of $A_{\mu}$. For all $x, y \in A_{\mu}, t \in(0,1]$, we have $A(x) \geq \mu$ and $A(y) \geq \mu$. Hence, $x, y \in A_{t}^{(\lambda, \mu)}$. If $(x y)_{t} \in A \cap B$, then $x \in B_{t}^{(\lambda, \mu)}$ or $y \in B_{t}^{(\lambda, \mu)}$, since $B$ is a $(\lambda, \mu)$-fuzzy prime ideal of $A_{\mu}$. So $x \in A_{t}^{(\lambda, \mu)} \cap B_{t}^{(\lambda, \mu)}=(A \cap B)_{t}^{(\lambda, \mu)}$, or $y \in A_{t}^{(\lambda, \mu)} \cap B_{t}^{(\lambda, \mu)}=(A \cap B)_{t}^{(\lambda, \mu)}$. It follows that $A \cap B$ is a $(\lambda, \mu)$-fuzzy prime ideal of $A_{\mu}$.

Similarly, we have the following theorem.

Theorem 20 Let $A$ be $a(\lambda, \mu)$-fuzzy ideal of $R$ such that $A_{\mu} \neq \emptyset$, and let $B$ be $a(\lambda, \mu)$-fuzzy semiprime (fuzzy primary, fuzzy semiprimary) ideal of $A_{\mu}$. Then $A \cap B$ is a $(\lambda, \mu)$-fuzzy semiprime (fuzzy primary, fuzzy semiprimary) ideal of $A_{\mu}$.

The following theorem gives the relation between a $(\lambda, \mu)$-fuzzy prime ideal with its preimage under a ring homomorphism.

Lemma 2 Let $f: R \rightarrow R^{\prime}$ be a homomorphism of rings, and let $B$ be a $(\lambda, \mu)$-fuzzy subring of $R^{\prime}$. Then for all $t \in(0,1], f^{-1}(B)_{t}^{(\lambda, \mu)}=f^{-1}\left(B_{t}^{(\lambda, \mu)}\right)$.

Theorem 21 Let $f: R \rightarrow R^{\prime}$ be a homomorphism of rings, and let $B$ be a $(\lambda, \mu)$-fuzzy prime ideal of $R^{\prime}$. Then $f^{-1}(B)$ is a $(\lambda, \mu)$-fuzzy prime ideal of $R$.

Proof From Theorem 9, $f^{-1}(B)$ is a $(\lambda, \mu)$-fuzzy ideal of $R$. Let $x, y \in R$ and $t \in(0,1]$. If $(x y)_{t} \in f^{-1}(B)$, then $(f(x) f(y))_{t} \in B$. Considering $B$ is a $(\lambda, \mu)$-fuzzy prime ideal of $R^{\prime}$, we have $f(x) \in B_{t}^{(\lambda, \mu)}$ or $f(y) \in B_{t}^{(\lambda, \mu)}$. Hence $x \in f^{-1}(B)_{t}^{(\lambda, \mu)}$ or $y \in f^{-1}(B)_{t}^{(\lambda, \mu)}$ from Lemma 2. It follows that $f^{-1}(B)$ is a $(\lambda, \mu)$-fuzzy prime ideal of $R$.

Similarly, we can obtain corresponding conclusions about a $(\lambda, \mu)$-fuzzy semiprime ideal, a $(\lambda, \mu)$-fuzzy primary ideal and a $(\lambda, \mu)$-fuzzy semiprimary ideal. But in general, the homomorphism image $f(A)$ of a $(\lambda, \mu)$-fuzzy prime ideal $A$ of $R$ may not be $(\lambda, \mu)$-fuzzy prime even if $f$ is a surjective homomorphism.

\section{Conclusion}

In this paper, we proposed the concept of a $(\lambda, \mu)$-fuzzy ideal which can be regarded as the generalization of a common fuzzy ideal introduced by Liu [11]. In the meantime, we also proposed several concepts of various $(\lambda, \mu)$-fuzzy ideals such as a $(\lambda, \mu)$-fuzzy prime ideal and a $(\lambda, \mu)$-fuzzy primary ideal, and then we characterized their properties and obtained several equivalence conditions of a $(\lambda, \mu)$-fuzzy prime ideal and a $(\lambda, \mu)$-fuzzy primary ideal. 


\section{Acknowledgements}

This work was supported by the National Natural Science Foundation of China (No. 71140008).

Received: 24 April 2012 Accepted: 3 September 2012 Published: 19 September 2012

\section{References}

1. Zadeh, LA: Fuzzy sets. Inf. Control 8, 338-353 (1965)

2. Rosenfeld, A: Fuzzy groups. J. Math. Anal. Appl. 35, 512-517 (1971)

3. Liu, W: Fuzzy invariant subgroups and fuzzy ideals. Fuzzy Sets Syst. 8, 133-139 (1982)

4. Dixit, VN, Kumar, R, Ajmal, N: Fuzzy ideals and fuzzy prime ideals of a ring. Fuzzy Sets Syst. 44, 127-138 (1990)

5. Kumar, R: Fuzzy semiprimary ideals of rings. Fuzzy Sets Syst. 42, 263-272 (1991)

6. Malik, DS, Mordeson, N: Fuzzy prime ideal of a ring. Fuzzy Sets Syst. 37, 93-98 (1990)

7. Bhakat, SK, Das, P: On the definitions of fuzzy subgroup. Fuzzy Sets Syst. 51, 235-241 (1992)

8. Bhakat, SK, Das, P: Fuzzy subrings and ideals redefined. Fuzzy Sets Syst. 81, 383-393 (1996)

9. Yuan, X, Zhang, C, Ren, Y: Generalized fuzzy groups and many-valued implications. Fuzzy Sets Syst. 138, 205-211 (2003)

10. Yao, B: $(\lambda, \mu)$-fuzzy subrings and $(\lambda, \mu)$-fuzzy ideals. J. Fuzzy Math. 15(4), 981-987 (2007)

11. Ming, PP, Ming, LY: Fuzzy topology 1: Neighbourhood structure of a fuzzy point and Moore-Smith cover. J. Math. Anal. Appl. 76, 571-579 (1980)

12. Bhakat, SK, Das, P: ( $\in, \in \vee q)$-level subset. Fuzzy Sets Syst. 103, 529-533 (1999)

doi:10.1186/1029-242X-2012-203

Cite this article as: Yao: Fuzzy prime ideals redefined. Journal of Inequalities and Applications 2012 2012:203.

\section{Submit your manuscript to a SpringerOpen ${ }^{\circ}$ journal and benefit from:}

- Convenient online submission

- Rigorous peer review

Immediate publication on acceptance

- Open access: articles freely available online

- High visibility within the field

- Retaining the copyright to your article 